\title{
Clinical and Microbiological Aspects of Chemotherapeutic Agents Used According to the Specific Plaque Hypothesis
}

\begin{abstract}
WALTER J. LOESCHE
Certain forms of dental decay and periodontal disease appear to be due to specific bacterial infections following overgrowth of certain indigenous plaque bacteria, i.e., the specific plaque hypothesis. If so, then antimicrobial treatment based on a diagnosis of elevated levels or proportions of these organisms should be considered. Such treatment cannot be administered according to concepts of the non-specific plaque hypothesis. A treatment philosophy is presented which is based upon considerations long established in medical infections following overgrowth of certain indigenous plaque bacteria, i.e., the specific plaque hypomicrobial to the site of the infection for periods long enough to suppress or destroy the pathogenic agent. Examples of this treatment philosophy are given.
\end{abstract}

University of Michigan, School of Dentistry, and School of Medicine, Ann Arbor, Michigan 48109

J Dent Res 58(12):2404-2412, December 1979

\section{Introduction.}

Bacteria are an essential component in both dental decay and periodontal disease. Their precise role has not been defined, but would include acid production in dental decay and the elaboration of a variety of low and high molecular weight products that can act as tissue irritants and antigens in periodontal disease. The dominant opinion in clinical dentistry has been that all plaque bacteria contribute to these dental diseases and that treatment should be directed toward plaque elimination or suppression. This opinion, which I shall call the nonspecific plaque hypothesis (NSPH) ${ }^{20}$ omits the need for a bacteriological diagnosis, and has led to the development of treatment and prophylactic strategies that rely extensively upon mechanical procedures to control plaque accumulations. Chemotherapeutic agents, while possible under the NSPH, in practice, when used daily in an effort to control plaque in all people, cannot com-

This research was supported by NIH grants DE-03423, DE-03011, and DE-02731. pare with the safety of mechanical procedures.

In effect, the universal open-ended treatment schedule of the NSPH is prejudicial against chemotherapeutic agents, because with usage all chemical agents invariably give rise to undesirable side effects or staining of the teeth. ${ }^{20}$

But what if the NSPH is not true or is only partially true? Would this open up new treatment and prophylactic strategies that would involve chemotherapeutic agents? In 1960 , Keyes ${ }^{15}$ demonstrated that at least one type of dental decay in hamsters was due to a transmissible Streptococcus mutans infection. Human isolates of $S$. mutans will cause a sucrose-dependent infection in several animal models that in many respects resembles human dental decay. ${ }^{16}$ Subsequently, human isolates of Actinomyces viscosus, ${ }^{14}$ Bacteroides (Capnocytophaga) ochracéus, ${ }^{12}$ Bacteroides melaninogenicus, ${ }^{25}$ and others have been shown to cause dental diseases in various animal models. Other investigations in humans have shown that plaques removed from diseased sites have characteristic microbial profiles that differ in significant ways from plaques removed from nondiseased sites in the same mouth or in different mouths. $8,21,24,28,31$ These unique microbial profiles raise the distinct possibility that the observed flora are contributing in a specific way to the concurrent disease process. If this is true, treatment should be directed toward their elimination or suppression. These recent observations revive an old concept in dentistry, namely, that some or all of dental decay and periodontal disease are due to specific bacterial infections. If so, then a specific plaque hypothesis ( $\mathrm{SPH}$ ) can be formulated which offers treatment strategies that can accommodate chemotherapeutic agents. ${ }^{20}$ Some aspects of these strategies will be briefly reviewed by means of answering several questions pertinent to antimicrobial treatment. 
Who should be treated? - The $\mathrm{SPH}$ is selective, since not everyone who forms plaque qualifies for treatment. In fact, the plaque must be diagnosed as odontopathic based upon precise clinical and bacteriological criteria before treatment can begin. The choice of agents will, to some extent, be based upon this diagnosis, and the treatment endpoint will be clinical healing and/or reduction of the odontopathic flora.

Which antimicrobial should be used? Chemotherapeutic agents should be chosen that have a spectrum of activity against the odontopathic flora. Since these flora are complex, first consideration should be given to the choice of a broad-spectrum antimicrobial. This might seem contradictory in a treatment philosophy based on microbial specificity but, as will be described later, the improvement that results from treatment according to the SPH comes about when the treatment stops and the dentogingival surfaces are repopulated by a nondisease-associated flora. Considerations such as substantivity, taste, and stability contribute to the choice of an agent. Chlorhexidine's remarkable antiplaque effect reflected its ability to adsorb to the dentogingival surfaces and then to be eluted in an active form. ${ }^{29}$ This substantivity of chlorhexidine led us to test it in patients with gingivitis. Fluorides also have the ability to be substantive in the mouth and can penetrate enamel, especially white spot lesions. ${ }^{4}$ For this reason, we chose fluorides as our antimicrobial agent in the treatment of rampant caries in children. Both of these agents are stable. However, neither has an agreeable taste, which presents problems concerning patient acceptability, particularly when used in an unsupervised manner. We had placed a premium upon taste in our earlier investigations and for this reason chose kanamycin as our antimicrobial agent. ${ }^{22}$ As will be described, however, kanamycin proved to be unsuitable in preventing certain carious lesions and was eventually replaced by fluoride.

How should the antimicrobial agent be delivered? - The SPH requires that the antimicrobial achieve an immediate result, i.e., elimination and/or suppression of the suspected odontopathic organism(s). Accordingly, the agent has to be delivered in effective dosages to the sites of infection. If topical agents are used, there is little possibility that the agent will have an effect on periodontitis or established carious lesions, since there is no way that ordinary topical delivery systems such as dentifrices, gels, mouthrinses, or lozenges can bring the agent to the site of infection. Topical agents will have access to coronal tooth surfaces so that a resolution of new carious lesions or gingivitis would seem possible. However, this is not an automatic occurrence as illustrated in the following examples. When high doses of fluoride were needed to reduce decay, the chlorhexidine had to be delivered to the gingival crevice by means of flossing in order to reduce a bleeding gingivitis. The agent should be delivered to the site of infection, i.e., the dentogingival surfaces, and not necessarily to the whole mouth. The dosages used when treating according to the SPH have to be high enough to cause a change in the odontopathic flora. Thus the dosages employed may be higher than are customarily used in dental treatment entrenched upon the tenets of the NSPH. Prescription levels of fluoride that were in excess of the low dosages employed in public health fluoride programs were used to reduce new decay in the rampant caries patients.

How long should treatment with the antimicrobial agent last? - The SPH dictates a short term usage of the antimicrobial agent. The strategy is to disrupt the existing disease flora to the extent that the proportion of odontopathogens surviving is either zero or so low that they are at a disadvantage in the recolonization process, thereby allowing a different and, presumably, a nonpathogenic flora to re-establish. This is the basic premise in the use of antimicrobials in medicine, where nonspecific agents such as penicillin cause dramatic cures in such specific infections as syphilis and streptococcal sore throats. Thus the healing response is dependent upon a short duration of treatment. We have arbitrarily chosen a one-week treatment period with fluoride in rampant caries children and two weeks of chlorhexidine treatment in gingivitis patients. The eventual length of treatment, as well as dosage levels, will have to be empiricaliy determined so as to find the optimal benefit-to-risk ratio which is associated with a clinical improvement 
and/or cure.

How should treatment efficacy be measured? - Treatment efficacy according to the SPH is measured in terms of disease resolution. In the case of dental decay, this would be an immediate reduction in the proportions or levels of specific cariogenic organisms that in time would be associated with a reduction in new carious lesions. In the case of gingivitis, this would be a reduction in periodontopathic organisms that would be coincidental with cessation of gingival bleeding.

Treatment of gingivitis with chlorhexidine. - Chlorhexidine has a remarkable ability to reduce plaque formation in vivo and thereby prevent gingivitis in the experimental gingivitis model. ${ }^{18,19}$ As such, chlorhexidine would appear to be the aggent of choice for plaque control and the treatment of gingivitis. However, daily usage of chlorhexidine, as would be advocated by the NSPH, causes in most people an aesthetically unacceptable stain, ${ }^{19,20}$ which precludes its marketability as an over-thecounter oral antimicrobial agent. We chose to evaluate chlorhexidine in a treatment schedule dictated by the SPH, namely, a short intensive application of gel to the dentogingival surfaces of patients with preexisting gingivitis. In the U.S., there is no Federal Drug Administration (FDA) approval for a chlorhexidine preparation in this manner. We obtained this approval after animal and human volunteer studies demonstrated no mucosal irritation or shifts in the bacterial flora which could be considered undesirable. In fact, the bacterial monitoring employed in these safety studies illustrated the types of bacterial shifts that one would hope would occur with an effective chemotherapeutic agent (Table 1). The proportions of $B$. melaninogenicus (these studies were performed prior to the now recognized need to subspeciate these black pigmented colonies) and $A$. viscosus decreased as a result of the two-week treatment period, and remained significantly suppressed compared to pretreatment values and during the four-week observation period. Since there are considerable animal and human data which associate both these species with periodontal disease, ${ }^{14,31}$ their suppression was interpreted as being beneficial. In fact, in these volunteers chosen from laboratory and clinical personnel, there was a significant reduction in the number of bleeding sites in the post-treatment period compared to their pretreatment numbers. No organisms that were resistant to $0.02 \%$ chlorhexidine were encountered at any time; however, low proportions of plaque organisms were already resistant to $0.002 \%$ chlorhexidine prior to treatment. These organisms included $B$. ochraceus, a periodontopathic organism in germfree rats, ${ }^{12}$ which is present in high proportions in diseased pockets found in juvenile periodontitis patients, ${ }^{28}$ and Streptococcus sanguis, an early colonizer of the tooth and contributor to plaque formation. ${ }^{8}$ The chlorhexidine treatment was selected for both organisms. However, the significant increase in proportions of $B$. ochraceus observed immediately after treatment was not sustained, and as the dentogingival surface was recolonized, the proportions of this organism significantly declined (Table 1). The apparent advantage conferred upon this organism by chlorhexidine treatment did not give $B$. ochraceus any predominance in the returning plaque flora. However, if the treatment had continued indefinitely, as advocated by the NSPH, then overgrowth of this potentially periodontopathic organism might have occurred, as has been reported in dog studies. ${ }^{10}$ (Incidentally, we use $0.002 \%$ chlorhexidine medium as a selective medium for $B$. ochraceus. [Syed and Loesche, unpublished data.]) However, $S$. sanguis, the other selected organism, became established in the returning plaque flora, an observation noted in other studies. $^{5,13,23}$ The selection of $S$. sanguis is beneficial as far as we can presently assess, since this organism seems to be present in elevated levels and proportions in non-diseaseassociated plaques (Loesche and Syed, unpublished data). At no time in this or subsequent investigations have we observed an increase in yeast as a result of chlorhexidine treatment.

The clinical testing of $0.2 \%$ chlorhexidine gel and a $0.05 \%$ quinine gel (placebo) was performed in young adults of both sexes who had pre-existing gingivitis manifesting itself clinically in at least five papillary sites that bled when a Stimudent ${ }^{R}$ was inserted interproximally. This method of diagnosing gingivitis was incorporated into a papillary bleeding score (PBS) that was used to monitor the clinical efficacy of the gel treat- 
TABLE 1

EFFECT OF 0.2\% CHLORHEXIDINE (CHX) GEL TREATMENT ON PROPORTIONS OF CERTAIN CULTIVABLE ORGANISMS REMOVED FROM A SINGLE GINGIVAL SITE IN ADULTS (15 subjects)

\begin{tabular}{|c|c|c|c|c|}
\hline & $\begin{array}{c}\text { Pre- } \\
\text { treatment }\end{array}$ & zero week & $\begin{array}{l}\text { Post-treatment } \\
\text { two weeks }\end{array}$ & four weeks \\
\hline Total Count $\times 10^{4}$ & $2030 \pm 820 \longleftrightarrow$ & $170 \pm 71 \longleftrightarrow$ & $1700 \pm 725$ & $1200 \pm 730$ \\
\hline \multicolumn{5}{|l|}{$\begin{array}{l}\% \text { organisms } \\
\text { resistant to }\end{array}$} \\
\hline $0.02 \% \mathrm{Chx}$ & None Detected & ND & ND & ND \\
\hline $0.002 \% \mathrm{Chx}$ & $0.4 \pm 0.2$ & $14.5 \pm 8.6$ & $3.7 \pm 1.4$ & $3.1 \pm 1.1$ \\
\hline $\begin{array}{l}\text { \% Bacteroides } \\
\text { melaninogenicus }\end{array}$ & $3.6 \pm 1.5$ & $3.2 \pm 1.1 \longleftrightarrow$ & $0.9 \pm 0.5$ & $0.6 \pm 0.5$ \\
\hline \% B. ochraceus & $0.8 \pm 0.3$ & $2.7 \pm 0.6$ & \pm 0.5 & $0.4 \pm 0.1$ \\
\hline$\%$ S. sanguis & $6.9 \pm 3.1$ & $15.3 \pm 7.1$ & $15.3 \pm 6.3$ & $23.8 \pm 6.0$ \\
\hline$\% A$. viscosus & $1.5 \pm 0.7$ & $0.2 \pm 0.1$ & $0.01 \pm 0.01$ & \\
\hline \% A. naeslundii & $3.6 \pm 2.6$ & $1.9 \pm 0.8$ & $5.3 \pm 3.1$ & \\
\hline
\end{tabular}

Differences between values connected by arrows are significant, $p<0.05$ paired t test.

Values within box are significantly different from all other values in row.

ments. The PBS criteria and their relationship to the gingivitis index ${ }^{18}$ are shown in Table 2. The PBS attempts to obtain accurate scoring of the clinical condition by having two examiners present for each examination and concurring on the score. Essentially, the PBS expands the GI = 2 score into three easily recognized clinical conditions, thereby increasing the sensitivity of the clinical observations concerned with

\section{TABLE 2}

PAPILLARY BLEEDING SCORE (PBS)

\begin{tabular}{|c|c|c|}
\hline $\mathrm{PBS}=0$ & $\begin{array}{l}\text { healthy gingiva; no bleeding } \\
\text { upon insertion of Stim-u-dent } \\
\text { interproximally }\end{array}$ & $\mathrm{GI}=0$ \\
\hline $\mathrm{PBS}=1$ & $\begin{array}{l}\text { edematous, reddened gingiva; } \\
\text { no bleeding upon insertion of } \\
\text { Stim-u-dent interproximally }\end{array}$ & $\mathrm{GI}=1$ \\
\hline $\mathrm{PBS}=2$ & $\begin{array}{l}\text { bleeding without flow upon } \\
\text { insertion of Stim-u-dent } \\
\text { interproximally }\end{array}$ & $\mathrm{GI}=2$ \\
\hline $\mathrm{PBS}=3$ & $\begin{array}{l}\text { bleeding with flow along } \\
\text { gingival margin upon insertion } \\
\text { of Stim-u-dent interproximally }\end{array}$ & $\mathrm{GI}=2$ \\
\hline $\mathrm{PBS}=4$ & $\begin{array}{l}\text { copious bleeding upon } \\
\text { insertion of Stim-u-dent } \\
\text { interproximally }\end{array}$ & $G I=2$ \\
\hline PBS $=5$ & $\begin{array}{l}\text { severe inflammation, marked } \\
\text { redness and edema; tendency } \\
\text { to spontaneous bleeding }\end{array}$ & $\mathrm{GI}=3$ \\
\hline
\end{tabular}

Consensus - two clinical examiners must agree. gingival bleeding. The PBS is determined on all papillae anterior to the second molars and omits readings on the buccal and lingual gingival margins. These latter sites rarely are involved in the initial stages of gingivitis, and the low scores derived from them in most clinical scoring systems dilute the more meaningful data that can be obtained from the dental papillae. Muihlemann and Soni have described a sulcular bleeding index (SBI), which is essentially the same as the PBS except that they used a probe to elicit any bleeding and did not obtain the consensus of two examiners. ${ }^{27}$ They have shown their SBI to be more sensitive than the GI in monitoring gingival changes, a finding with which we agree.

The most sensitive indicator of gingival improvement was a reduction in the number of papillary sites that bleed with a flow, i.e., PBS $\geq 3$. A two-week unsupervised use of chlorhexidine or quinine gel applied to the dentogingival surfaces in trays, twice a day for five minutes, for each of 14 days was associated with a 43 percent reduction in the number of these sites (Table 3). The improvement associated with the chlorhexidine treatment persisted somewhat longer than that seen with quinine. However, the difference in treatment effect between the two groups was minimal and in agreement with results reported by others. 1,30 Flotra and his colleagues ${ }^{7}$ had observed that chlorhexidine was not effective when 
TABLE 3

EFFECT OF CHLORHEXIDINE AND QUININE GELS EITHER ALONE OR IN COMBINATION WITH FLOSSING OR DENTAL PROPHYLAXIS ON NUMBER OF BLEEDING SITES $\geq 3$ PER PATIENT

\begin{tabular}{|c|c|c|c|}
\hline $\begin{array}{l}\text { two week } \\
\text { treatment } \\
\text { period }\end{array}$ & $\begin{array}{l}\text { Percen } \\
\text { zero wk }\end{array}$ & $\begin{array}{c}\text { reduction } \mathrm{f} \\
\text { Values } \\
\text { two wk }\end{array}$ & $\begin{array}{l}\text { m initial } \\
\text { four wk }\end{array}$ \\
\hline $\begin{array}{l}\text { Chlorhexidine } \\
\text { Chx + prophy } \\
\text { Chx + flossing }\end{array}$ & $\begin{array}{l}43 \pm 8 \\
62 \pm 9 \\
85 \pm 5\end{array}$ & $\begin{array}{l}44 \pm 8 \\
38 \pm 11 \\
71 \pm 6\end{array}$ & $\begin{array}{l}28 \pm 9 \\
18 \pm 9 \\
72 \pm 7 \\
\end{array}$ \\
\hline $\begin{array}{l}\text { Quinine } \\
\text { Quinine }+ \\
\text { prophy } \\
\text { Quinine }+ \\
\text { flossing }\end{array}$ & $43 \pm 12$ & $\begin{array}{l}39 \pm 11 \\
27 \pm 13\end{array}$ & $19 \pm 16$ \\
\hline Flossing & $68 \pm 7^{*}$ & $27 \pm 16$ & $7 \pm 13$ \\
\hline & \multicolumn{3}{|c|}{$\begin{array}{l}\text { Values in box are significantly } \\
\text { different ( } p \leq 0.05 \text { independent } \\
t \text { test) from all other values in } \\
\text { column except those marked by } \\
\text { an asterisk. }\end{array}$} \\
\hline
\end{tabular}

pockets or sulci greater than $3 \mathrm{~mm}$ in depth were present, the argument being that the mode of delivery did not bring the chlorhexidine into the pocket or sulcus. We modified our treatment protocol to include groups which received a dental prophylaxis midway between the two-week treatment period and groups who were instructed to apply the gel as instructed and then to floss the gel between the teeth. The flossing group was comprised of patients who stated that they routinely flossed their teeth. The dental prophylaxis resulted in an immediate improvement in the chlorhexidine group, but this effect did not persist (Table 3). Flossing was associated with an 85 percent reduction in bleeding sites in the chlorhexidine group and a 64 percent reduction in the quinine group. The reduction observed in the chlorhexidine-flossing group was significantly greater than that observed in the quinine-flossing group. Evidently the flossing had brought the chlorhexidine to the site of infection and had altered the flora in a fashion that promoted gingival healing in the post-treatment period. However, much of the clinical improvement in the immediate post-treatment period could be due to the mechanical debriding action of the flossing. This was demonstrated by having another group of patients floss for a two-week period and then observing their PBS over a four-week, noflossing interval. As shown in Table 3, flossing alone was very effective in reducing the number of bleeding sites, but this treatment effect disappeared when the flossing was discontinued. Thus, the initial improvement in the chlorhexidine-flossing group was due to both the chlorhexidine and the flossing, while the residual benefits most likely reflected the re-establishment of a non-disease-associated flora, as predicted by the SPH.

The above experiments illustrate some of the treatment outcomes predicted by NSPH because of its staining characteristics and potential to select for a possible periodontopathic organism such as $B$. ochraceus. However, while flossing does not appear to eliminate the periodontopathic flora, it can suppress it when performed frequently, if not daily. This daily mechanical debridement causes an obvious clinical improvement and, because of its apparent safety, would be the treatment of choice for gingivitis. However, daily flossing is difficult for most people to maintain. Short-term chlorhexidine treatment, when brought in effective dosages to the site of infection, i.e., by flossing, can cause a sustained clinical improvement, presumably by the establishment of a nondisease-associated flora. Thus, in some instances, treatment of gingivitis as if it were a specific infection may be indicated. Subsequent clinical trials will be necessary to determine the relative efficacy, safety, and cost of either treatment in various populations.

Treatment of rampant caries individuals with fluoride. - Fluoride has long been associated with caries reduction due to mechanisms involving fortification of the tooth enamel by filling voids in the apatite lattice and by formation of fluorapatite crystals. 4,26 However, fluoride is able to inhibit a number of bacterial enzymes, including enolase, an enzyme involved with substrate uptake and energy metabolism. ${ }^{9}$ We came to evaluate fluoride as an antimicrobial agent in the treatment of $S$. mutans infections in rampant caries children after an initial experiment with kanamycin led us to hypothesize that white spot lesion served as a reservoir for cariogenic 
organisms. ${ }^{22}$ We wanted an antimicrobial agent which was capable of penetrating the white spot, and fluoride was known to do this. ${ }^{4}$ The clinical observations which led us to formulate the white spot hypothesis are shown in Table 4. A 5 percent kanamycin or placebo gel was given to patients who arrived at the dental office with ten or more carious surfaces. The patients were instructed how to apply the gels in an applicator tray to the dentogingival surfaces twice a day for one week prior to the placement of all necessary dental restorations and for one week after the placement of these restorations. The treatment was unsupervised at home. The patients were seen at three-month recall intervals when the plaque was sampled and new cavitation recorded. Within the first eight to ten months after treatment, the number of new carious lesions was greater in the kanamycin-treated patients (Table 4). This caused us to stop the entry of new patients into the study and to closely monitor the existing kanamycin and placebo patients. Over the next one to two years, the kanamycin patients developed few new lesions, whereas the placebo patients continued to develop new lesions. When the data were eventually summarized, the kanamycin treatment was associated with a net caries reduction of about 45 percent compared to the placebo treatment. This left us with the need to explain how kanamycin caused an acceleration in decay and then a quiet period, in which the placebo group caught up and surpassed the kanamycin group in terms of new decayed surfaces. This was done by postulating the white spot lesion as a reservoir for cariogenic organisms.

The white spot hypothesis. - The white spot hypothesis is conceivable only in the context of bacterial specificity in the carious process. It states that certain cariogenic organisms, i.e., S. mutans, are within the white spot. When an antimicrobial agent is delivered in effective concentrations to the tooth surface, it will nonspecifically reduce the tooth surface flora. If the agent cannot penetrate the white spot, whether because of ionic charge or occlusion of the channels within the white spot by bacterial cells, these organisms within the white spot will survive (Fig. 1). When the treatment ceases, the survivors within the white spot
TABLE 4

CUMULATIVE CARIES INCREMENT ${ }^{\mathrm{a}}$ FOUND

AT RECALL FOLLOWING EITHER KANAMYCIN (kan) OR PLACEBO (pla) GEL TREATMENT

\begin{tabular}{|c|c|c|c|c|c|}
\hline \multirow[b]{3}{*}{$\mathrm{Gel}$} & \multicolumn{5}{|c|}{ Tooth Surface } \\
\hline & \multicolumn{2}{|c|}{ Occlusal } & \multicolumn{2}{|c|}{ Approximal } & \multirow{2}{*}{$\begin{array}{l}\text { Smooth } \\
\text { kan pla }\end{array}$} \\
\hline & kan & pla & kan & pla & \\
\hline \multicolumn{6}{|c|}{ Months } \\
\hline \multirow{2}{*}{\multicolumn{6}{|c|}{$\begin{array}{l}\text { After } \\
\text { Treatment }\end{array}$}} \\
\hline & & & & & \\
\hline $5-7$ & 0 & 7 & 0 & 0 & $\begin{array}{ll}0 & 0\end{array}$ \\
\hline $8-10$ & 12 & 7 & 6 & 0 & 3 \\
\hline $11-13$ & $13(1)^{b}$ & $13(6)$ & 6 & 6 & 3 \\
\hline $14-16$ & $18(5)$ & $22(9)$ & $7(1)$ & $7(1)$ & $5(2)$ \\
\hline $17-20$ & 18 & $24(2)$ & 7 & $11(4)$ & 3 \\
\hline$>20$ & 19 & $29(5)$ & $8(1)$ & 11 & 35 \\
\hline
\end{tabular}

a)Diagnosis by cavitation, b) figures in parentheses are new cavitations detected at recall examination, c) data adapted from ref. 22.

are able to grow out from the lesion and become dominant in the newly established plaque flora (Fig. 2). If these surviving organisms include $S$. mutans, then the carious process is accelerated. On tooth surfaces which did not contain white spots, the kanamycin treatment led to a reduction or elimination of $S$. mutans in the returning plaque flora, since most of the organisms which repopulate the tooth surface in this instance come from the saliva, 8 and the salivary levels of $S$. mutans are relatively low. Thus, the effective treatment of the tooth surfaces with kanamycin will accelerate decay in the clinically undetectable incipient lesions and retard or eliminate decay on other surfaces. The placebo treatment is without effect, and the decay will continue in these patients at the normal rate. The white spot hypothesis predicts that an antimicrobial agent capable of penetrating the white spot will prevent decay from being accelerated in these lesions. The failure of kanamycin to penetrate would not appear to be due to its molecular weight, since it is a relatively small molecule. But, as with chlorhexidine, it has a net positive charge, which could cause it to bind at the tooth surface. Fluoride, which is known to penetrate the ename $1^{4}$ and to be elevated in arrested lesions, ${ }^{17}$ has a negative charge. Fluoride, according to the model, would penetrate the lesion and kill the bacteria 
in situ (Fig. 1). When the treatment is stopped, the tooth surface over the lesion would be repopulated by any survivors left on the tooth surface, by spread of organisms from adjacent teeth and by those organisms in the bathing saliva which have the ability to adhere to the tooth surfaces. This sequence of events would not lead to a selection of $S$. mutans in the newly formed plaque.

The white spot hypothesis permits speculation concerning the role that dead bacteria left within the white spot can have on future caries development at that site. Years ago, Enright et al. ${ }^{6}$ showed that the enamel white spots were resistant to acid solubilization in experiments in which extracted teeth containing them were placed in an acid solution. This was because the white spots were impregnated with acid insoluble organic material. The white spot model states that the organic material would include bacterial remnants. Thus, if bacteria present in the white spots can be killed in situ, their remains could serve as an organic sealant which might effectively plug the formerly acid-soluble channels within the lesion, thereby aborting the further development of the lesion. Organisms like $S$. mutans could accumulate on the surface of the lesion without any enhancement of caries, a situation observed when xerostomia patients were treated daily with high levels of fluoride. ${ }^{3}$

Morphological verification of bacteria in white spot lesions was recently provided by Brannstrom et al. ${ }^{2}$ Electron photomicro. graphs led them to conclude that: "The presence of bacteria in small lacunae in the enamel prism, as well as deep in the enamel under white spot lesions, together with pores in the enamel surface, would seem to indicate that bacteria may penetrate the enamel at an early stage."

Clinical testing of the white spot hypothesis. - We tested the white spot hypothesis by conducting two double blind studies on rampant caries children with fluoride as the antimicrobial agent. According to the model, if sufficient fluoride can be delivered to the depths of the white spot lesion, a reduction of new decay would be seen in the first year after treatment. In the first investigation, a fluoride mouthrinse containing $1 \%$ fluoride ${ }^{+}$(containing

\footnotetext{
${ }^{+}$Iradicav, Janar Co., Grand Rapids, MI
}

$0.6 \%$ fluoride as APF and $0.4 \%$ stannous fluoride) was used for three weeks unsupervised at home following complete restoration of diagnosed lesions. Results one year after the mouthrinse revealed that the fluoride patients had 1.4 new carious surfaces per patient compared to 0.4 in a group receiving a placebo mouthrinse (Loesche and Bradbury, unpublished data). After two years, the amount of decay in the placebo group caught up to and surpassed that found in the fluoride group, so that the fluoride group now had 34 percent less decay. It was the results of the kanamycin study repeated. Did this mean that the model was wrong or that the mouthrinse delivery system for a three-week period was an inadequate delivery system? In a concurrent investigation, a $1.2 \%$ fluoride gel $\$$ (neutral $\mathrm{NaF}$ ) was given to young children using applicator trays twice a day for a one-week period, prior to and after the placement of the necessary dental restorations. The treatment was unsupervised at home and was repeated for one week at approximately six-month intervals. After one year there was approximately a 50 percent reduction in new carious surfaces in the fluoride group compared to the placebo group (Table 5). This would seem to indicate that effective levels of fluoride were delivered to the white spots so as to abort their development and that the gel delivery system was superior to a mouthrinse in

\section{TABLE 5}

EFFECT OF UNSUPERVISED TOPICAL NaF GEL OR PLACEBO GEL TREATMENT ON SUBSEQUENT CARIES EXPERIENCE IN RAMPANT CARIES CHILDREN* ONE YEAR LATER

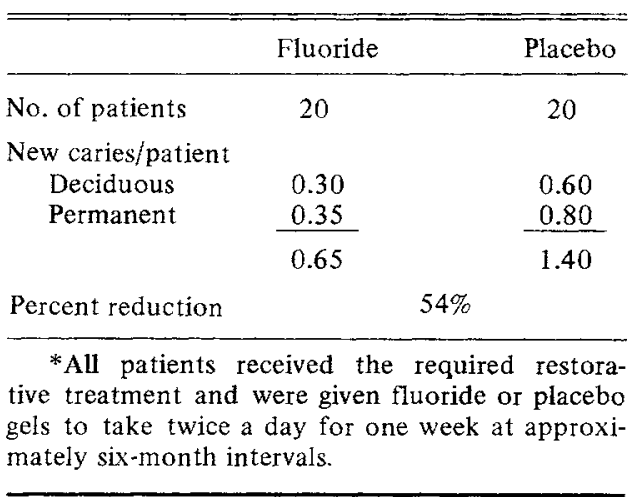

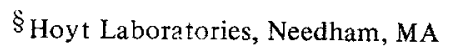


accomplishing this end. This is not surprising since a mouthrinse is distributed over the entire oral surfaces for short periods of time, whereas the gel confined the agents to the dentogingival surfaces for five minutes, if it was used as directed. The levels of fluoride in both the mouthrinse and gel were prescription levels considerably higher than those recommended for caries control under NSPH. In the gel study, about $6 \mathrm{ml}$ of gel, or $60 \mathrm{mg}$ of fluoride, was placed in the trays with each application. If 10 to 20 percent of the gel was swallowed, the patient received 6 to $12 \mathrm{mg}$ of systemic fluoride each time he used the trays. This in itself is not harmful for a seven-day period ${ }^{11}$ and could be considered beneficial in terms of fortifying the unerupted teeth. The use of this level of fluoride is justified only in patients who have high levels of active decay and who are being treated in accordance with the concepts of the SPH.

\section{Acknowledgments.}

Procter and Gamble allowed us to refer to their FDA file in regard to our obtaining an IND number from the FDA for chlorhexidine. Janar Co., Grand Rapids, MI, and Hoyt Lab., Needham, MA, provided the fluoride agents and their respective placebos. David Bradbury, Thomas Pink, and Natalie Grossman provided clinical measurements, while Salam Syed and Merritt Walker coordinated the bacteriological aspects of the investigation.

\section{REFERENCES}

1. BUIN, J. M. and STRAHAN, J. D.: The Effect of a $1 \%$ Chlorhexidine Gel in the Initial Therapy of Chronic Periodontal Disease, J Periodont 49:469-474, 1978.

2. BRANNSTROM, M.; FRISKOPP, J.; ISACSSON, G.; and WICTORIN, L.: Experimental Caries in Young Human Permanent Teeth Implanted in Removable Dentures, Arch Oral Biol 22:571-578, 1977.

3. BROWN, L. R.; DREIZEN, S.; and HANDLER, R.: Effects of Selected Caries Preventive Regimens on Microbial Changes Following Irradiation-induced Xerostomia in Cancer Patients, Proceedings, Microbial Aspects of Dental Caries, Stiles, Loesche and O'Brien, eds., Sp. Supp. Microbiol Abs 275-290, 1976.

4. BRUDEVOLD, F. and McCANN, H. G.: Fluoride and Caries Control. Mechanism of Action. The Science of Nutrition and its
Application in Clinical Dentistry, Philadelphia: Saunders, 1966, pp. 331-347.

5. EMILSON, C. G. and FORNELL, J. Effect of Toothbrushing with Chlorhexidine Gel on Salivary Microflora, Oral Hygiene, and Caries, Scand J Dent Res 84:308-319, 1976.

6. ENRIGHT, I. J.; FRIESELL, H. E.; and TRESCHER, M. O.: Studies on the Cause and Nature of Dental Caries, $J$ Dent Res 12:759-851, 1932.

7. FLOTRA, L.; GJERMO, P.; R $\emptyset$ LLA, G.; and WAERHAUG, J.: A Four-month Study on the Effect of Chlorhexidine Mouthwashes on 50 Soldiers, Scand J Dent Res 80:10-15, 1972.

8. GIBBONS, R. J. and VAN HOUTE, J.: On the Formation of Dental Plaque, $J$ Periodont 44:347-360, 1973.

9. HAMILTON, I. R.: Effects of Fluoride on Enzymatic Regulation of Bacterial Carbohydrate Metabolism. In: Cariostatic Mechanisms of Fluorides, Caries Res 11 (Suppl.): 321-327, 1977.

10. HAMP, L. E. and EMILSON, C. G.: Some Effects of Chlorhexidine on the Plaque Flora of the Beagle Dog, $J$ Periodont Res 8:(Suppl.) $28-40,1973$.

11. HODGE, H. C.: Fluoride Toxicity. In: Fluorides and Dental Caries, Springfield, IL: E. Newbrun, C. C. Thomas, ed., 1975, pp. 138-146.

12. IRVING, J. T.; SOCRANSKY, S. S.; NEWMAN, M. G.; and SAVIT, E.: Periodontal Destruction Induced by Capnocytophaga in Gnotobiotic Rats, IADR Progr \& Abst 55:No. 783, 1976.

13. JORDAN, H. V. and DePAOLA, P.: Effect of Prolonged Topical Application of Vancomycin on Human Oral Streptococcus mutans Populations, Arch Oral Biol 22:193-200, 1977.

14. JORDAN, H. V.; KEYES, P. H.; and BELLOCK, B.: Periodontal Lesions in Hamsters and Gnotobiotic Rats Infected with Actinomyces of Human Origin, $J$ Periodont Res 7:21-25, 1972.

15. KEYES, P. H.: The Infections and Transmissible Nature of Experimental Dental Caries: Findings and Implications, Arch Oral Biol 1:304-310, 1960.

16. KEYES, P. H.: Present and Future Measures for Dental Caries Control, JADA 79:13951404, 1969.

17. LEVINE, R. S.: Distribution of Fluoride in Active and Arrested Carious Lesions in Dentin, $J$ Dent Res 51:1025-1029, 1972.

18. LOE, H.: Human Research Model for the Production and Prevention of Gingivitis, $J$ Dent Res 50:256-265, 1971.

19. LÖE, H. and SCHIÖTT, C. R.: The Effect of Mouthrinses and Topical Application Chlorhexidine on the Development of Dental 
Plaque and Gingivitis in Man, $J$ Periodont Res 5:79-84, 1970.

20. LOESCHE, W. J.: Chemotherapy of Dental Plaque Infections, Oral Sci Rev 9:63-105, 1976.

21. LOESCHE, W, J, and SYED, S. A.: Bacteriology of Human Experimental Gingivitis. II. Effect of Plaque and Gingivitis Score, Infect and Immun 21:830-839, 1978.

22. LOESCHE, W. J.; BRADBURY, D. R.; and WOOLFOLK, M. P.: Reduction of Dental Decay in Rampant Caries Individuals Following Short Kanamycin Treatment, $J$ Dent Res 56:254-265, 1977.

23. LOESCHE, W. J.; MURRAY, R. J.; and MELLBERG, J. R.: The Effect of Topical Fluoride on Percentages of Streptococcus mutans and Streptococcus sanguis in Interproximal Plaque, Caries Res 7:283-296, 1973.

24. LOESCHE, W. J.; ROWAN, J.; STRAFFON, L. H.; and LOOS, P. J.: The Association of Streptococcus mutans with Human Dental Decay, Infect and Immunol 11:1252-1260, 1975 .

25. MACDONALD, J. B.; SOCRANSKY, S. S.; GIBBONS, R. J.: Aspects of the Pathogenesis of Mixed Anaerobic Infections of Mucous Membranes, J Dent Res 42:529-534, 1963.
26. MYERS, H. M.: The Mechanism of the Anticaries Action of Fluoride Ion. In: Fluorides and Dental Caries, E. Newbrun, ed., Springfield, IL: C. C. Thomas, 1975, pp. 99-112.

27. MUHLEMANN, H. R. and SON, S.: Gingival Sulcus Bleeding - A Leading Symptom in Initial Gingivitis, Helv Odont Acta 15: 107$113,1971$.

28. NEWMAN, M. G. and SOCRANSKY, S. S.: Predominant Cultivable Microbiota in Periodontosis, $J$ Periodont Res 12:120-128, 1977.

29. RøLLA, G.; LOE, H.; and SCHIOTT, R.: Retention of Chlorhexidine in Human Oral Cavity, Arch Oral Biol 16:1109-1115, 1970.

30. RUSSELL, B. C. and BAY, L. M.: Oral Use of Chlorhexidine Gluconate Toothpaste in Epileptic Children, Scand $J$ Dent Res 86: $52-57,1978$.

31. SLOTS, J.: The Predominant Cultivable Microflora of Advanced Periodontitis, Scand J Dent Res 85:114-121, 1977. 